\title{
Phytosociological Database of Non-Forest Vegetation in Croatia
}

\author{
Zvjezdana Stancic
}

\begin{abstract}
Phytosociological investigations based on the Braun-Blanquet approach were begun in Croatia, by Ivo Horvat and Stjepan Horvatic in the first half of the 20th century. They have had many successors, most of whom were active in the period between 1960 and 1990. A considerable amount of data, both from the literature and those newly collected in the field, has been accumulated. Due to the application of this data to scientific purposes, the construction of a phytosociological database has been in progress for more than ten years. TURBOVEG software is used for storage of the data. So far, over 5,700 relevés from the territory of Croatia have been collected. Regarding the coverage of specific vegetation types, the best represented are: grassland, marshland, water, halophilous coastal vegetation and the vegetation of trampled habitats. For these vegetation types, almost all available data have been collected. This report describes the available content in the Phytosociological Database of Non-Forest Vegetation in Croatia (GIVD ID EU-HR001).
\end{abstract}

Keywords: Croatia; phytosociology; relevé; vegetation database.

\section{Phytosociological Database of Non-Forest Vegetation in Croatia}

Scope: Non-forest vegetation in Croatia. Number of relevés: grassland vegetation $n=3,586$; marshland vegetation: $n=841$; water vegetation $n=247$;

halophilous coastal vegetation $n=483$;

vegetation of trampled habitats $n=283$;

ruderal vegetation $n=184$ (in preparation); forest vegetation $n=104$ (in preparation).

Status: ongoing capture

Period: 1927-2009

Database manager(s): Zvjezdana Stancic (zvjezdana.stancic@gfv.hr, zvjezdana.stancic@kr.t-com.hr)

Owner: Zvjezdana Stancic (private)

Web address: [NA]

Availability: free upon request

Online upload: no

Online search: no

Database format(s): TURBOVEG

Export format(s): TURBOVEG

Publication: Stancic, Z. 2008: Phytosociological database of non-forest vegetation of Croatia. In: Chytrý, M. (ed.), Using phytosociological data to address ecological questions. Abstracts and Excursion Guides. 17th International Workshop European Vegetation Survey, 128. Czech Republic, Brno, Masaryk University: 1-5 May 2008.

Plot type(s): normal plots

Non-overlapping plots: 5,728

Plot-size range: $0.4-60 \mathrm{~m}^{2}$

Total plot observations: 5,728

Estimate of existing plots: 12,000

Completeness: $48 \%$

Countries: HR: $100.0 \%$

Forest: [NA] — Non-forest: [NA]

Guilds: all vascular plants: $100 \%$

Environmental data: altitude: $24 \%$; slope aspect: $33 \%$; slope inclination: $28 \%$; soil $\mathrm{pH}: 11 \%$

Performance measure(s): cover: $100 \%$

Geographic localisation: GPS coordinates (precision $25 \mathrm{~m}$ or less): $20 \%$; point coordinates less precise than GPS, up to $1 \mathrm{~km}$ : $58 \%$; small grid (not coarser than $10 \mathrm{~km}$ ): $22 \%$

Sampling periods: 1920-1929: 1.0\%; 1930-1939: 4.0\%; 1950-1959: 2.0\%; 1960-1969: 14.0\%; 1970-1979: 17.0\%; 1980-1989: 8.0\%; 1990-1999: 19.0\%; 2000-2009: 14.0\%; unknown: $21.0 \%$

Information as of 2012-07-12; further details and future updates available from http://www.givd.info/ID/EU-HR-001

Zvjezdana Stancic (zvjezdana.stancic@gfv.hr, zvjezdana.stancic@kr.t-com.hr)

Faculty of Geotechnical Engineering, University of Zagreb, Hallerova aleja 7, 42000 Varazdin, CROATIA 